\title{
Small Bowel Volvulus In Adult: A Review Of Literature
}

\author{
D. C. Shyam and R. C. Shyam
}

\begin{abstract}
A Small bowel volvulus is a rare entity where the small bowel loop twists around its own mesentery. These twisting may produce a mechanical obstruction, vascular compromise, or both. In North America and Western Europe, the annual incidence of small bowel volvulus is 1.7 to 5.7 cases per 100,000 , but much higher rates of 24 to 60 cases per 100,000 have been observed in Africa, Asia, the Middle East, and India. Small bowel volvulus can be classified as primary and secondary subtypes. In primary variety, there is no known cause whereas secondary variety occurs due to the presence of predisposing lesions, either congenital or acquired of which postoperative adhesion is the most common responsible factor. The most commonly accepted explanation for the initiation of primary small bowel volvulus is the abnormal bowel peristalsis generated due to the sudden overfilling of the empty bowel loop with a large portion of meal after prolong fasting. Secondary small bowel volvulus is commonly seen in the elderly population with mixed gender distribution. There are no specific clinical features or laboratory findings and a high index of suspicion is required to reach a diagnosis. Abdominal pain is the most common presenting feature of small bowel volvulus. CT scan is the investigation of choice and a whirl sign is the characteristic finding of bowel volvulus. Urgent Surgical exploration is the treatment of choice to prevent complications due to vascular compromise. Although small bowel volvulus is rare, it has a mortality rate between $9 \%$ and $35 \%$, which can reach up to $100 \%$ if associated with bowel necrosis.
\end{abstract}

Index Terms - Volvulus, small bowel Volvulus, whirl sign, bowel necrosis.

\section{INTRODUCTION}

A Small bowel volvulus (SBV) is a rare condition [1] where the small bowel (SB) twists around its own mesenteric axis [2]. The incidence of SBV varies between $0.00001 \%$ to $0.19 \%$ [3]. Volvulus is more commonly seen in children compared to adults [4]. The SBV is broadly divided into primary and secondary variety based on the etiology [5]. Due to its non-specific clinical features, the preoperative diagnosis of SBV is difficult [6]. In terms of management, CT scan is the investigation of choice, and surgery is the mainstay of the treatment. Mortality in SBV was reported even up to $100 \%$ when associated with necrosis of the bowel [7].

\section{HISTORY}

The terminology volvulus evolved from the Latin word "volvere" which means "to roll or twist" [8] - [10]. The first description of volvulus was mentioned in papyrus Ebers, dated $1550 \mathrm{BC}$ as a "natural course of twisting of a segment of the alimentary tract led to either spontaneous resolution or rotting of the intestines". Hippocrates was the first to treat volvulus by injecting a large quantity of air through the anus and by inserting a 10 digit $(22 \mathrm{~cm})$ long suppository [9]. The first report of volvulus in western literature was published in 1841 and Atherton for the first time described laparotomy as the treatment of volvulus in 1883. In 1947, Brusgaard challenged surgical intervention for volvulus because he successfully managed 91 patients of sigmoid volvulus without peritonitis by decompression via sigmoidoscopy and rectal tube placement. He reported a mortality rate of $14 \%$ but in subsequent follow up he observed $90 \%$ recurrence and the mortality rate increased to $40 \%$. This might be the reason why endoscopic decompression is still not the main treatment modality [10].

\section{DEFINITION}

SBV is a condition where the bowel loops coiled around the axis of its own mesentery [4]. Midgut volvulus occurs when the bowel loop twist more than $180^{\circ}$ around its own mesenteric vascular pedicle [8]. This coiling can lead to partial or complete mechanical obstruction [11] with or without the interference of its blood circulation [12]. Volvulus of the SB is rare and most commonly it involves the sigmoid colon [7], [13].

\section{INCIDENCE}

The annual incidence of SBV is 3.5 cases [14]. The SBV accounts for 1 to $25 \%$ of small bowel obstruction (SBO) [1], [5], [7], [11], [15] and 1.7 to $27 \%$ of all intestinal obstruction (IO) [4], [13] - [17].

The incidence of SBV is high in Middle Eastern, Asian, central African countries [18] and Finland [14] compared to the western countries [18], [19]. However, some of the literature from Asia reported that the incidence of SBV is low in Japan and Taiwan [18]. In Western countries, the incidence of SBV is 1.5 to $5.7 / 100000$ adults [20] of which 3 to $6 \%$ of patients presented with IO [3], and 24 to 60/100000 adults in Africa and the Asian continent [20] of which 20 to $50 \%$ of the patients presented with IO [3].

In the West, secondary small bowel volvulus (SSBV) is more common whereas in Africa and Asia continent primary small bowel volvulus (PSBV) accounts for most of the cases [20]. In western countries, the prevalence of PSBV and SSBV is 10 to $22 \%$ and 70 to $90 \%$ respectively however, in the regions of the central part of Africa, Middle Eastern 
countries, Afghanistan, Iran, and the Indian subcontinent, PSBV accounts for 31 to $100 \%$ [14].

Volvulus accounts for $25 \%$ of SBO in pregnant women. Volvulus in the mid and late pregnancy can occur in any part of the gastrointestinal tract (GIT) of which sigmoid colon is the most common site. SBV is rare whereas sigmoid volvulus is the most common cause of intestinal obstruction during pregnancy accounting for $25 \%$ to $44 \%$ [21].

\section{Age And Gender}

SBV can occur at any age [14] but it is found to be quite rare in adults [14], [18]. According to the statistics, PSBV is commonly seen in children and young adults, whereas SSBV is prevalent mainly in adults [14]. Literature has reported that SSBV is prevalent much higher in the elderly group ranging from 60 to 90 years [4], [8], [14], [22].

Brinda et al. stated that SSBV is common in 6 to 8 decades and it is quite uncommon before the age of 40 years old [15]. Furthermore, it has also been reported by Birnbaum et al. that SSBV is usually encountered in the age group of 40 to 90 years old [23].

In terms of gender distribution, literature wise there is a bit of disagreement: [24];

- SBV is found to be slightly predominant in females [4],

- Chia-Hsiang Li et al. have found SBV to be prevalent in males with a ratio of $2: 1$ [22];

- Xiao-Fei Shen et al. reported that SSBV occurs in older patients and it has been found that there is equality with respect to the gender distribution [6].

\section{ClassificAtion}

SBV can be classified as primary and secondary variety, where PSBV occurs in a normal abdominal cavity with no underlying anatomical abnormalities or predisposing factors and SSBV occurs due to the presence of predisposing lesions, either congenital or acquired [5], [7], [14], [25].

Inukai et al. classified SSBV based on the cause of the torsion (Table I) [26].

\section{TABLE I: CLASSIFICATION OF SSBV}

\begin{tabular}{ll}
\hline Type A & $\begin{array}{l}\text { Most common variety. Torsion initiated due to } \\
\text { SB tumor or SB intussusceptions }\end{array}$ \\
\hline \multirow{2}{*}{ Type B } & $\begin{array}{l}\text { Bowel twist around the fixed point created due to } \\
\text { the adhesion of the SB to the abdominal wall or a } \\
\text { strangulated hernia } \\
\text { Rarest variety. It is due to the adhesion of the } \\
\text { proximal and distal bowel or the adhesion } \\
\text { between the bowel and the mesocolon. Peristaltic } \\
\text { waves in the intestinal between those points lead } \\
\text { to torsion }\end{array}$ \\
\hline \hline
\end{tabular}

\section{ETIOLOGY}

The incidence of PSBV is $48 \%$, where there is no known cause [15] whereas SSBV is multifactorial of which postoperative adhesion (74\%) [27] is the most common cause [4], [14], [15], [17], [18], [24].

Other congenital and acquired factors responsible are:
Malrotation of midgut [15], [16], Meckel's diverticulum [9], [15], [16], [25], ileal atresia, mesenteric cyst, ascariasis, bands, meconium ileus, enteroenterostomy [15], [25], Jejunal diverticulum [15], [28], adhesions [9], [15], [16], [25], tumors [9], [15], [16], Leiomyoma [15], [16], [25], pregnancy during third trimester [9], [15], [16], [25], internal hernias [16], [25], mesenteric lymph nodes, endometriosis, tuberculosis, aneurysm, hematomas [16], previous stoma, small bowel diverticula [9], chylolymphatic mesenteric cyst, intestinal duplication, ovarian cyst, meconium pseudocyst, congenital mesenteric defect, persistent omphalocele cyst, ovarian cyst, simultaneous pancreas and kidney transplantation, paraduodenal hernia [15], Surgeries [25], laparoscopic appendectomy [15], complications following laparoscopic surgery [9] and anaesthesia drugs causing intestinal bloating and fast decompression, bowel handling, patient positioning [15], orthodontic brace [29].

T. Patial et al. mentioned low socioeconomic status, diabetic autonomic neuropathy, and parasitic infestation as the risk factors for SBV [16].

\section{PATHOPHYSIOLOGY}

The difference in the pathophysiology of SBV with the large bowel Volvulus is that it twists around the dorsal mesentery [30]. Volvulus can lead to mechanical intestinal obstruction due to the abnormal twisting of the bowel loops around its own mesentery. The clinical manifestations are due to the narrowing or obliteration of the bowel lumen or cutting off of the blood supply, or both [14]. If the bowel distension increases or if the mesentery twisted further, blood flow to the involved intestine can become compromised leading to ischemia [9], [11] and tissue hypoxia [11]. Obstruction and strangulation frequently crop up and progress quickly to gangrene as the mesenteric twist tends to be very tight leading to early vascular involvement [20]. The sequelae of that will steer to intestinal obstruction, bowel gangrene, perforation peritonitis [1], [7]. If an extensive segment of the loop is involved, then a large volume of blood and plasma are extravasated into the intestinal wall and lumen. Due to the breach of the mucosa, the gut bacteria enter into the lymphatics and capillaries leading to septic shock, multiorgan failure [11], and death [7], [11].

Causes of primary SBV are multifactorial [14]. The etiology is not known, but the factors which may be responsible are stasis of the bowel contents, a long and narrow band-like mesentery, rapid changes in the intraabdominal pressure, and hyperperistalsis [31]. Dietary factor along with prolonging fasting is considered to be one of the factors leading to the development of the SBV [18], as it is most commonly seen after breaking fast during the month of Ramadan [14], [18], or during winters and spring season in north-western Ethiopia [32]. The incidence of SBV also seems to increase during the summer months in underdeveloped rural areas when marriages and other feasts are mostly celebrated [14], and also in rural Nepal during the months which are filled with festivals and feasts [15]. Jr. J. H. Duke and M. S. Yah reported that the risk of SBV increased to 9-10 fold during the holy month of Ramadan and it was related to abrupt changes in the dietary intake [33].

Some individuals have longer mesentery and narrow 
mesenteric root which would allow abnormal mobility of the entire SB or of a segment of it. This abnormal bowel mobility occurs due to the sudden overfilling of the empty bowel with a single large bulky fibre rich diet after prolong fasting [16]. The sudden passage of a large bulky meal into the proximal jejunum makes it heavier causing it to drift inferiorly or towards the left lower quadrant due to the lack of resistance in the pelvis. As a result of that, the distal jejunum and ileum are forced in a clockwise rotation into the right lower quadrant swaying to the torsion of the mesentery. Young age and muscular abdominal wall aid in twisting of the bowel loop [5], [34]. Birnbaum et al. stated that the bowel peristalsis initiates by the sudden overload of empty bowel by large and poorly digested voluminous meals [23].

The suggested mechanism for SSBV involves obstruction of a SB loop at two fixed points by any of the predisposing factors. As the loop fills with food particles, peristalsis causes it to twist around its mesentery [25].

The factors responsible for PSBV such as a long SB, broad mesentery free of fat, firm abdominal muscle restricting the bowel movement to the coronal plane and a large amount of high fiber diet after prolong starvation are challenged based on the following basis; Not all primary SBV rotate clockwise, It does not account for further twists after the first $360^{\circ}$; Solid or semi-digested not always found in the bowel but instead whole grain or maize husks, and Females with a lax abdominal wall also develop SBV in Nepal. According to the author intrinsic motility of the gut could be the cause of the volvulus, and the points in favour of that are:

1) In one of the studies, they have found that 12 patients developed PSBV within a few hours of drinking a large quantity of local beer which contains a high concentration of serotonin (5-hydroxytryptamine) which increases gut motility.

2) One report mentioned that in diabetes, change of bowel tone and peristaltic activity due to diabetic autonomic neuropathy may be the cause of volvulus.

3) Chronic parasitic infestation of the bowel is common in areas where PSBV is frequent and also parasitism alter bowel motility.

The author further stated that there must be a complex mechanism mediated by chemicals in the food or by lowgrade enteritis due to worms, protozoa or bacteria that alter the bowel motility and initiate a volvulus [35].

\section{Clinical Features}

Diagnosis of SBV is difficult due to its non-specific symptoms [6], [17], [36]. A high index of suspicion is required, and an early diagnosis is essential to avoid mesenteric ischemia and gangrene [7], [22].

SBV presents either acutely (89\%) due to acute vascular insufficiency or peritonitis, or else with vague symptoms of abdominal pain, nausea, vomiting, abdominal distension, and a decrease in flatus production [9].

The most common presenting feature of SBV is abdominal pain [14], [18], [24]. The severity of the pain is directly related to the level of vascular occlusion and not to the level of intestinal obstruction, and the intensity of the pain does not correspond with clinical findings, as only $26 \%$ of the patients present with peritoneal signs [37]. Common clinical manifestations in order of frequency are abdominal pain (94 to $100 \%$ ), nausea or vomiting ( 83 to $100 \%$ ), distension (55 to $100 \%$ ), peritoneal irritation (14 to $26 \%$ ). They may present all together or may occur in any combinations [14].

Due to its non-specific clinical feature, various authors suggested patterns of clinical features which can hint us towards the early diagnosis of SBV like: [5].

Central abdominal pain resistant to narcotic analgesia

Colicky abdominal pain with a comparatively painless interval, vomiting, abdominal tenderness, and position of relief i.e. lying on one side or knee-elbow position which relaxes the tension on mesentery resulting in relief of pain [36].

- Recent history of intermittent, recurring abdominal pain that occurs after the ingestion of a meal [23].

- Recurrent, intermittent periumbilical or epigastric pain occurring after the ingestion of a meal with severity out of proportion to clinical examination [16].

- Periumbilical pain doesn't subside with analgesics and associated with nausea, vomiting, and abdominal distension, and peritonism [15].

- Epigastric or periumbilical pain for several days and sudden onset of clinical symptoms and signs of SBO without previous abdominal surgery and the severity of pain is out of proportion to clinical examination [38].

- Upper abdominal pain after meals, alternating diarrhoea and constipation, tenderness, and weight loss [6].

Clinical presentation of midgut volvulus is usually nonspecific unless an acute SBO with signs of peritonitis and/or systemic inflammatory responses occurs [6]. Clinical features suggestive of peritoneal irritation although an indication for urgent Laparotomy is nonspecific and present in an only quarter of patients with SBV [17].

Constant severe abdominal pain, associated with tenderness, muscle guarding, tachycardia, fever, leucocytosis along with radiological findings such as pneumatosis, portal venous gas, pneumoperitoneum, submucosal haemorrhage or free fluid on CT scan are the indications of bowel ischemia and necessitate for early surgical intervention [39].

Because of its vague and nonspecific nature, it mimics irritable bowel syndrome, peptic ulcer disease, pancreatic disease, Biliary disease, psychiatric disease [2], [9], acute appendicitis [8].

\section{InVESTIGATIONS}

No clinical features, deranged laboratory, and abnormal radiological findings are typical of SBV [34].

\section{A. Laboratory investigations}

The laboratory findings are neither sensitive nor specific for the diagnosis of SBV [37].

Usually, a patient with suspected IO will have Hypokalemic, hypochloremic metabolic alkalosis if he develops severe vomiting. Elevated blood urea nitrogen levels, hemoglobin, and hematocrit levels are consistent with dehydration. Intestinal bacteria translocation into the bloodstream, which can lead to systemic inflammatory response syndrome or sepsis, gives raised white blood cells. Metabolic acidosis with increased serum lactate indicates 
bowel ischemia [40].

J. Santin-Rivero et al. stated that leucocytosis is present in $66 \%$ of the patients with mesenteric torsion but leucocytes count of over $10,000 / \mathrm{cm} 3$ was invariably present in $100 \%$ of the patients who developed bowel necrosis [37]. C. L. N. Tsang et al. reported that among biochemical markers, an elevated lactate level can indicate mesenteric ischemia or necrosis but this too can be normal in the presence of necrosis [1].

\section{B. Plain Abdominal Radiograph (PAR)}

PAR is rarely helpful due to its nonspecific findings [18], [30], [41]. It can also, neither distinguish viable from a nonviable bowel nor identify volvulus as a cause [12].

PAR can demonstrate nonspecific signs of SBO i.e. dilated SB loops with air-fluid level and occasionally pneumatosis intestinalis and thumbprinting impression which are the signs suggestive of intestinal ischemia [8].

Rarely PAR will show dilated bowel loops with a spiral nebula in the mid-abdomen [5], or mass effect in the midabdominal region and whirled appearance of the bowel [42], or distended stomach, duodenum and SB proximal to the transition point with a collapse appearance or lack of gas shadow in the distal bowel loops [30], which are all suggestive of SBV.

\section{Fluoroscopy imaging of upper GIT}

Upper git fluoroscopic is mainly advised when malrotation is suspected in children [30] and if SBV is present it will show the characteristic corkscrew appearance [30], [41]. However, this test is time-consuming and many a time poorly tolerated when associated with SBV [9] and do not allow rapid diagnosis [12].

\section{Ultrasonography (USG)}

USG is sensitive in infants where the IO is due to SBV, but it is not sensitive in adults because it is operators dependent. If it is successful, it will show either a whirlpool sign or classic barber pole sign [9].

$\mathrm{X}$. Li et al. reported that USG can only reveal distended bowel loops with reverse peristalsis, and it cannot pick any specific findings or the etiology that leads to IO [18] whereas E. P. Burke stated that it is often possible to detect the whirlpool sign [8].

Parcos et al. first described the whirlpool sign in USG and reported 89 to $100 \%$ sensitivity in diagnosing SBV. They also stated that the absence of a whirl sign doesn't rule out SBV [15].

The sensitivity, specificity and positive predictive value of the whirl sign for SBV are 92\% [5], [8], 100\% [5], [8], and $100 \%$ [5] respectively but the major disadvantage of sonography is that it is operator dependent hence chances of miss diagnosis is very high resulting in disastrous consequences [5].

Peterson et al. reported that USG cannot detect the abnormal position of the bowel but occasionally it can detect the abnormal position of the superior mesenteric artery (SMA) and Superior mesenteric vein (SMV), and probably because of this USG is not a recommended investigation [41].

\section{E. CECT Abdomen scan (CT scan)}

CT scan is the investigation of choice for SBV [5], [8] with a sensitivity of 60 to $100 \%$ [5], [6], [12] and specificity of 90 to $95 \%$ [12]. The bowel wrapping around the SMA creates the typical whirl pattern [43] which is the characteristic feature of SBV in CT scan [5], [12]. It is present in $75 \%$ of cases with SBV [12].

In 1981, Fisher observed that the bowel loops with the mesentery and the surrounding soft tissue encircling the twisted mesenteric vessels, and coined the term "whirl sign". Twisting of $\geq 90^{\circ}$ of bowel loop has $64 \%$ sensitivity in diagnosing SBV but in most of the cases, the twisting is at least $180^{\circ}$. Other signs suggesting of SBV are: the abnormal positioning of the SMA and SMV reflecting the twisting of the mesenteric vessels and the Beak sign which is the fusiform tapering of compressed bowel loops at the site of twisting and the tip or the apex represent the point of rotation [2].

CT scan sensitivity depends on the length and orientation of the loop in relation to the imaging plane [15]. Volvulus is best appreciated when imaging is perpendicular to the axis of bowel rotation [30].

According to Jaramillo and Raval, CT scan picture of primary SBV are:

1) Thickening of bowel wall and mesentery and blunting of the valvulae conniventes due to venous and lymphatic obstruction;

2) Arterial obstruction indicated by thumbprint impression, pneumatosis intestinalis, and gas in the portal venous system;

3) Closed-loop obstruction;

4) Abnormal location of intestinal loops;

5) Twisting of the mesenteric root or Whirl sign due to the twisting of the mesentery [19].

M. Lepage-Saucier et al. observed three signs of SBV which are multiple transition points, transition points located $\leq 7 \mathrm{~cm}$ from the spine in the anteroposterior plane, and the whirl sign: swirl extending $\geq 180^{\circ}$ including both bowels and vessels around a fixed point of obstruction. The presence of any one of these signs confirms SBV with a sensitivity of $94 \%$ whereas the presence of all the three signs confirms SBV with $100 \%$ specificity [30].

Matsuki et al. interpret some CT scan finding which could help in preoperative diagnosis of SBV and those findings are $\mathrm{U}$ shaped configuration or radial distribution of the distended bowel, fluid-filled loops of the SB converging towards the point of torsion, a fusiform tapering loop of the SB or a beak sign in longitudinal section [34].

CT scan also shows $\mathrm{C}$ or $\mathrm{U}$ shaped bowel loops, radially arranged bowel loops around its mesenteric pedicle, two adjacent collapsed SB loops with the fecal shadow which is gas or solid material within the dilated bowel loop [12], [15]. Angiography can detect twisting in the mesenteric vessels, dilated SMV, prolonged contrast transit time but as it is a time consuming and invasive procedure, it is not routinely used in emergency conditions [6].

SB ischemia is a potentially fatal complication of SBV and CT imaging is useful in detecting bowel ischemia. Though bowel enhancement can be normal, increased, decreased, or absent making diagnosis challenging there are other signs which help in the diagnosis of bowel wall ischemia. These are bowel wall thickening, mesenteric edema, and ascites. An early but non-specific sign of bowel edema and inflammation 
is the target sign i.e. thickened bowel with an enhanced inner and outer layer, sandwiching a middle layer of low attenuation [12].

When the triad of high-grade bowel obstruction, presence of free fluid on CT, and absence of flatus on per abdominal examination is present then there is a high likelihood for the need for early laparotomy [9].

As the fact goes, volvulus is a surgical emergency, and delay in the early diagnosis carries a high mortality rate for both mother (6 to $20 \%$ ) and fetus (20 to $26 \%$ ). Imaging is essential for early and precise diagnosis of volvulus. The mean and the maximum dose of plain abdominal X-ray are $1.4 \mathrm{mGy}$ and $4.2 \mathrm{mGy}$ respectively and that of CT scan abdomen is $8 \mathrm{mGy}$ and $49 \mathrm{mGy}$ respectively. The Radiation exposure within the diagnostic ranges of $<50 \mathrm{mGy}$ is not associated with an increased incidence of congenital malformation in either humans or animals. Thus, both abdominal plain film and CT are safe for the fetus, with the former much safer. In terms of radiation exposure MRI is safer than a CT scan [21]. MRI is also extremely precise in the diagnosis of SBO and sometimes also showing the "whirlpool sign" [41]. In sum, plain abdominal film, MRI and/or ultrasound belong to the first choice, while CT can be chosen if necessary [21].

\section{TREATMENT}

SBV is a surgical emergency and warrants emergency surgical intervention to prevent complications [8]. Once SBV is suspected there is no room to consider for conservative treatment and immediate Laparotomy is indicated as volvulus with compromised vascular status carries a greater risk of gangrene [34]. The management of SBV consists of conservative and/or surgical measures. Though the main treatment of SBV is surgical exploration, other supportive measures are Intravenous fluid supplement, insertion of Ryle's Tube and self-retaining catheter, and venous thromboembolism prophylaxis [1].

Non-operative management though not recommended, is reserved for those who are unfit for surgery, but with a possible higher mortality rate [45].

Definitive diagnosis can only be made on exploratory Laparotomy [46]. The management of SBV with bowel gangrene is clear [5], [34] and that is, resection and primary anastomosis [34], [40] or stoma [46], but controversies arise when the bowel loop is viable [5]. Untwisting and the resulting mesenteric decompression are the crucial step to avoid bowel necrosis, therefore, preventing intestinal resection which ultimately leads to a better outcome [23]. The main controversy is whether to perform simple devolvulation or further augmentation with intestinal fixation or prophylactic bowel resection [1]. One school of thought advocates simple devolvulation whereas the other recommends resection-anastomosis even if there is no necrosis [5]. The extent of the bowel resection will be based on the length of the involved bowel segment [34].

On the table, it is essential to deliver the SB out of the abdominal cavity especially when there are many dilated bowel loops. Refraining from that may result in missing the cause of the obstruction and also the volvulus may be completely overlooked [47].
In the absence of necrosis, if the bowel loop appears to be edematous or congested the simple derotation, with or without fixation of the bowel can be considered, however, this procedure is associated with recurrence of SBV [5]. S. Islam et al. suggested that in PSBV fixation is recommended if resection is not performed because the recurrence rate is as high as $30 \%$ in patients who underwent simple devolvulation only [5]. On the other hand, another author suggested that if the bowel loop is viable a simple derotation is sufficient, as recurrence is not known with simple derotation. In this particular case report, the cause of the SBV is multiple giant ileojejunal diverticulosis [46].

$\mathrm{X}$. Li et al. stated that the patient status, anatomy of the SB, length of the involved bowel, and mesentery should be considered on the table. If no anatomical abnormalities then simple devolvulation without fixation should be done as the rate of recurrence is less whereas resection or fixation of the intestine should be considered depending on the surgeon's experience, long mesentery or any other associated anatomical abnormalities [18].

Enteropexia or intestinal adherence procedure are the procedures, developed to prevent recurrent IO due to adhesions. This procedure creates controlled adhesions that keep the intestines in a certain position to prevent volvulus or obstruction. In the well-known Noble procedure, the small bowel loops were folded in a zigzag manner every 15 to 20 $\mathrm{cm}$, starting 15 to $20 \mathrm{~cm}$ away from the duodenojejunal junction and leaving the ileocecal valve free. The bowel wall is sutured with catgut. In the McCarthy modification of Childs-Phillips procedure, instead of suturing the SB loops, the mesentery is joined by sutures in a $U$ shape perforating the mesentery proper 3 or $4 \mathrm{~cm}$ away from the mesenteric border of the bowel loops [37]. There are many techniques and variations hence it's solely the surgeon's decision based on his skills and experience.

X.-F. Shen et al. reported a case of SBV diagnosed preoperatively with a CT scan. On exploration, they found a jejunal diverticulum with adhesions as the cause of the volvulus. They untwisted the volvulus segment, performed adhesiolysis and excise the jejunal diverticulum. They further arranged the small bowel in step ladder pattern starting from $15 \mathrm{~cm}$ away from the Ligament of Treitz till 20 proximal to the ileocecal junction. The mesentery was fixed using interrupted sutures with 4-0 silk at a gap of every 1.2 to $2 \mathrm{~cm}$ leaving the folded part of the bowel loops. The sutures were placed $2-3 \mathrm{~cm}$ away from the mesenteric border and the whole arrangement was fixed with retroperitoneum instead of the peritoneum [6].

J. S. Bhullar et al. reported spontaneous resolution of SBV following the ingestion of oral contrast material. After initial evaluation in a 49 years old patient for IO, they advised a CT scan of the abdomen and pelvis to diagnose the cause of intestinal obstruction. CT scan revealed SBV with characteristic whirl sign. Within 1 hour of consuming oral contrast, the abdominal pain resolved followed by opening up of his bowel. They stated that possibly the osmotic nature of the contrast material initiated the peristalsis and along with the volume of the contrast, bowel loop detwisted and resumed its normal configuration [7].

The Laparoscopic approach was first used to deal with IO in 1991 by Bastug et al. They performed laparoscopic band 
release for intestinal obstruction caused by a single adhesive band [48].

Martin and Steele stated some favorable point for the laparoscopic approach:

1) Beneficial in patients who carry a high risk from pulmonary morbidity following laparotomy or wound complications.

2) Used as an adjunct to decompress or detwist the volvulus along with the standard resection.

3) May be used to performing non-resectional intervention such as suture pexy or colostomy tube placement.

4) Avoid pain and complications associated with a long laparotomy incision [49].

In spite of the upper hand, there are no clear guidelines for the laparoscopic approach in the treatment of SBV [26].

Valsdottier and Marks reported that minimal invasive surgery (MIS) is acceptable but it solely depends upon the surgeon's experience. Abdominal incision should be made carefully to avoid bowel injuries. Open technique such as Hasson cannula or optiview should be used preferably. Severe distension and signs of peritonitis should be taken as contraindication for MIS [13].

Kim et al. stated that if the cause of SBV is uncertain, then diagnostic laparoscopy is a prized option for making the definitive diagnosis and administering treatment [50].

$\mathrm{K}$. Inukai et al. reported that the laparoscopic approach is a better option than laparotomies in terms of outcome namely post-operative intestinal motility and duration of hospitalization. However laparoscopic surgery is not suitable in some cases due to the abnormal bowel position, limited space due to adhesions, and dilated bowel loops. The postoperative adhesions are especially very difficult to separate, and in that scenario, adhesiolysis should be done very cautiously and the option for conversion to Laparotomy should always be kept open [26].

In one of the articles, the author recommended laparoscopic surgery in a patient with suspected SBV due to the advantages of less invasive, faster recovery and from the diagnostic point of view in case of uncertain diagnosis [18].

\section{PROGNOSIS AND Mortality}

Diagnosis of SBV is very difficult to make due to its nonspecific clinical manifestations which ultimately leads to a major mishap [14], [18], [34]. Clinical judgement by experienced senior surgeons, clinical parameters (fever, tachycardia, peritoneal signs, bowel sounds) or laboratory tests (Haemoglobin concentration, hematocrit level, WBC counts, or electrolytes) is neither specific nor sensitive to differentiate simple or strangulated bowel obstruction [14]. Diagnostic imaging should not be considered in a sick patient with a rigid abdomen, as further delay will increase the likelihood of unnecessary morbidity and mortality [3].

Mortality in non-infarcted SBV varies from 5 to $35 \%$ [7], [12], [14], [15], [17], [18], [23] - [25], where the general consensus lies between 10 to $35 \%$ (11) and it reaches up to $100 \%$ if it is associated with necrosed bowel [7], [16], [17], [23], [25]. S. Islam et al. reported that the mortality rate due to SBV is as high as 42 to $67 \%$ [5].

High mortality is seen in patients with a history of abdominal surgery and cardiopathies [45].

In the elderly age group, the outcome uses to be lethal if associated with bowel necrosis and it can be as high as $90 \%$. The prognosis of PSBV is good because the majority of the patients are young hence more physiologically fit, and perforation is uncommon [34].

\section{REFERENCES}

[1] C. L. N. Tsang, C. T. Joseph, M. S. B. De Robles, S. Putnis, "Primary Small Bowel Volvulus: An Unusual Cause of Small Bowel Obstruction", Cureus, vol.11 (12), pp. 6465, 2019.

[2] A. Wright, S. Chong, J. Willatt, "Small bowel volvulus around caesarian section scar adhesions", Radiol Case Radiol., vol. 12(3), pp. 504-507, 2017.

[3] J. J. Leow, T. Huey, J. K. Low, "Primary adult midgut volvulus mimicking acute appendicitis: a case report and review of the literature", Int J Surg Case Rep., vol. 24, pp. 182-184, 2016.

[4] M. Kapadia, "Volvulus of the small bowel and colon", Clin Colon Rectal Surg., vol. 30(1), pp. 40-45, 2017.

[5] S. Islam, D. Hosein, D. Dan, V. Naraynsingh, "Volvulus of ileum: A rare cause of small bowel obstruction". BMJ Case Reports, vol. 19, 2016.

[6] X. F. Shen, W. X. Guan, K. Cao, H. Wang, J. F. Du, "Small bowel volvulus with jejunal diverticulum: Primary or secondary?", World J Gastroenterol, vol. 21(36), pp. 10480-10484, 2015.

[7] J. S. Bhullar, P. Papapetrou, G. Subhas, N. Gupta, S. Seman, V. K. Mittal, "Spontaneous Resolution of Primary Small Bowel Volvulus with Oral Contrast”, J Curr Surg, vol. 2(3), pp. 110-112, 2012.

[8] E. P. Burke, M. Saeed, M. Mahmood, C. Hayes, M. Salama, and I. Ahmed, "Whirl Sign and Midgut Volvulus: An Unusual Cause of an Acute Abdomen in an Adult Patient", Case Reports in Surgery, 2019.

[9] Z. M. Bauman, C. H. Evans, "Volvulus", Surg Clin North Am., vol. 98 (5), pp. 973-993, 2018

[10] D. Gingold, Z. Murrell, "Management of colonic volvulus", Clin Colon Rectal Surg., vol. 25, pp. 236-244, 2012.

[11] J. Klein, K. Baxstorm, S. Donnelly, et al. "A fatal twist: volvulus of the small intestine in a 46 year old woman", Case Rep Med. 2015; Article ID 391093:4.

[12] M. Takatsuka, M. A. Shah, B. Dharmarajah, R. Brightwell, N. Damani, "A rare case of adult primary jejunal volvulus", BMJ Case Rep. 2010; doi: 10.1136/bcr.09.2009.2242.

[13] E. Valsdottir, J. H. Marks, "Volvulus: small bowel and colon", Clin Colon Rectal Surg, vol. 21, pp. 91-93, 2008.

[14] A. Roggo, L. W. Ottinger, "Acute small bowel volvulus in adults. A sporadic form of strangulating intestinal obstruction", Ann Surg, vol. 216(2), pp. 135-141, 1992.

[15] M. A. Brinda, S. Manjunath, K. S. Balasubramanya, B. Nanjaiah, "An unusual case of small bowel volvulus", J Clin Diagn Res, vol. 9, pp. 8$10,2015$.

[16] T. Patial, S. Chaddha, N. Rathore, V. Thakur, "Small Bowel Volvulus: A Case Report”, Cureus, vol. 9(5), pp. 1281, 2017.

[17] P. G. Katis, S. M. Dias, "Volvulus: a rare twist on small- bowel obstruction" CMAJ, vol., 171 (7), pp. 728, 2004.

[18] X. Li, J. Zhang, B. Li, D. Yi, C. Zhang, N. Sun et al. "Diagnosis, treatment and prognosis of small bowel volvulus in adults: A monocentric summary of a rare small intestinal obstruction", PLoS ONE. 2017; 12(4). e 0175866. Doi:10.1371/journal.pone.0175866.

[19] D. Jaramillo, B. Raval, "CT diagnosis of primary small-bowel volvulus", AJR Am J Roentgenol, vol. 147(5), pp. 941-942, 1986.

[20] J. D. Wig, S. M. Bose, S. P. Kaushik, "Volvulus obstruction of the small andlarge bowels", J Postgrad Med, vol. 24, pp. 117-120, 1978.

[21] Q. Cong, X. Li, X. Ye, L. Sun, W. Jiang, Z. Han et al. "Small bowel volvulus in mid and late pregnancy: can early diagnosis be established to avoid catastrophic outcomes?", Int J Clin Exp Med, vol. 7, pp. 4538$4543,2014$.

[22] C. H. Li, C. H. Chen, J. W. Chou, "Intestinal obstruction caused by small bowel volvulus", Am J Med, vol.124, pp. 3-4, 2011.

[23] D. J. Birnbaum, E. Grègoire, P. Campan, J. Hardwigsen, Y. P. Le Treut, "Primary small bowel volvulus in adult", J Emerg Med, vol. 44, pp. 329-330, 2013.

[24] T. M. Coe, D. C. Chang, J. K. Sicklick, "Small bowel volvulus in the adult populace of the United States: results from a population-based study”, Am J Surg, vol. 210, pp. 201-210, 2015.

[25] U. Aydin, P. Yazici, H. Toz, C. Hoscoskun, A. Coker, "Management of small bowel volvulus in a patient with simultaneous pancreas-kidney 
transplantation a case report”, Journal of Medical Case Reports, vol. 1, pp. 106, 2007.

[26] K. Inukai, H. Kitagami, S. Uehara, et.al. "A rare case of secondary small bowel volvulus laparoscopically repositioned: literature review and classification", Surgical Case Reports, vol. 4(1), pp. 65, 2018.

[27] J. H. Ryu, H. J. Yang, G. Lee, Y. S. Lim, J. K. Kim, E. Ryoo et al. "Small bowel volvulus in adults: A case report", J Korean Soc Emerg med. vol. 13(3), pp. 373-376, 2002.

[28] M. Y. Hong, C. H. Chi, "Small-Intestinal Volvulus", N Engl J Med. vol. 365(4), pp. 358, 2011.

[29] T. Shepherd, A. Teo, R. Naunton-Morgan, "Orthodontic braces come back to bite: a novel presentation of a small bowel volvulus", BMJ Case Rep. 2017; 2017: bcr2017221152.

[30] M. Lepage-Saucier, A. Tang, J. S. Billiard, J. Murphy-Lavallée, L. Lepanto, "Small and large bowel volvulus: Clues to early recognition and complications", Eur J Radiol. vol. 74, pp. 60-66, 2010.

[31] Y. Kitano, K. Hashizume, M. Ohkura, "Segmental small-bowel volvulus not associated with malrotation in child hood", Pediatr Surg Int., vol. 10, pp. 335-338, 1995.

[32] K. Ghebrat, "Seasonal variation of primary small intestinal volvulus in North West Ethiopia", East Cent Afr J surg, vol. 6, pp. 87-90, 2001.

[33] J. H. Duke, M. S. Yar, "Primary small bowel volvulus. Cause and management", Arch. Surg.vol. 112(6), pp. 685-688, 1977.

[34] N. Ali, M. Mubarak, Y. Ali, "Primary volvulus of the small intestine", Ann Nigerian Med., vol. 7, pp. 86-89, 2013.

[35] I. O. McDonald, D. B. Hawker, "Small bowel volvulus: the commonest abdominal emergency in Nepal", Bristol Med Chir J, vol. 95, pp. 4-8, 1980.

[36] E. Christensen, "Volvulus of Small Bowel Diagnosed Pre-operatively", Br Med J., vol. 1, pp. 442, 1958.

[37] J. Santín-Rivero, E. Núñez-García, M. Aguirre-García, G. HagermanRuiz-Galindo, F. de la Vega-Gonzalaz, C. R. Moctezuma-Velasco, "Intestinal volvulus. Case report and a literature review", Cir Cir, vol. 83, pp. 522-526, 2015

[38] G. Papadimitriou, A. Marinis, A. Papakonstantinou, "Primary midgut volvulus in adults: report of two cases and review of the literature", $J$ Gastrointest Surg.vol. 15, pp. 1889-1892, 2011.

[39] B. W. C. Ferris, "Small bowel volvulus post gastric bypass", Int Surg J. vol. 6(2), pp. 590-592, 2019.

[40] P. G. Jackson, M. T. Raiji, "Evaluation and management of intestinal obstruction", Am Fam Physician, vol. 83(2), pp. 159-165, 2011.

[41] C. M. Peterson, J. S. Anderson, A. K. Hara, J. W. Carenza, C. O. Menias, "Volvulus of the gastrointestinal tract: appearances at multimodality imaging", RadioGraphics, vol. 29(5), pp. 1281-1293, 2009.

[42] P. J. Strouse, "Disorders of intestinal rotation and fixation ("malrotation")", Pediatr Radiol, vol. 34, pp. 837-851, 2004.

[43] S. M. Bernstein, P. D. Russ, "Midgut volvulus: a rare cause of acute abdomen in an adult patient', Am J Roentgenol, vol. 171(3), pp. 639641,1988

[44] P. E. Constanthin, M. Darouichi, "Volvulus of the small bowel due to malrotation during pregnancy: A case report", Journal of digestive disorders and diagnosis, vol. 1(3), pp. 34-40, 2017.

[45] B. Barakat, R. Pezzilli, "Volvulus of ileum: A rare case of small bowel obstruction and the effectiveness of abdominal ultrasound imaging for the diagnosis", Emergency Care Journal, vol. 15(2), pp. 8154, 2019.

[46] A. Khan, F. Khan, K. Rafique, S. Khan, A. Majid, K. Khan et al. "Small Gut Volvulus, A Rare Twist, In The Setting Of An Even Rarer Entity; Multiple Giant Jejuno Ileal Diverticula", J Ayub Med Coll Abbottabad, vol. 29(1), pp.147-149, 2017.

[47] C. H. Talbot, "Volvulus of the small intestine in adults", Gut, vol. 1, pp.76-80, 1960.

[48] T. M. S. Salama, "Laparoscopic Management of Acute Intestina Obstruction', Surgery Curr Res, vol. 7, pp. 290, 2017

[49] M. J. Martin, S. R. Steele, "Twists and turns: a practical approach to volvulus and intussuception", Scand J Surg, vol. 99, pp. 93-102, 2010

[50] K. H. Kim, M. C. Kim, S. H. Kim, K. J. Park, G. J. Jung, "Laparoscopic management of a primary small bowel volvulus: a case report", Surg Laparos Endosc Percutan Tech, vol. 17, pp. 335-338, 2007.

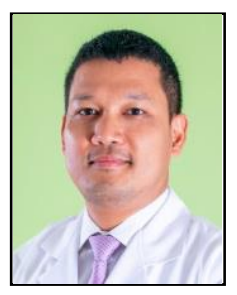

Dr. Devajit Chowlek Shyam is a General and Laparoscopic Surgeon from India. He completed his M.S (General Surgery) in 2007 and DNB (General Surgery) in 2013 from India. He has got 12 years of experience with him and currently working as a specialist, General Surgery in AsterCedars hospital, Dubai, UAE

His field of interest is Emergency Surgeries, Minimally Invasive Open Thyroidectomy, Esophageal surgery, Biliary and Pancreatic surgery, Upper GIT surgeries and Hernia surgeries. He has got over 10 publications in national and international journals, and few are accepted for publication.

Dr Shyam is a life member with the Association of Surgeons of India.

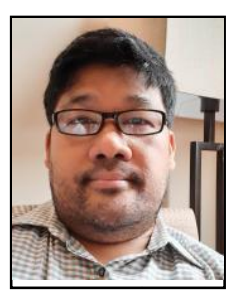

Dr. Ranjit Chowlek Shyam is a General and Laparoscopic surgeon with an experience of 15 years. He is currently holding the post of Assistant professor (Department of General Surgery) in Silchar Medical college and Hospital, Assam, India. His field of interest is Advance Laparoscopic Surgeries, Trauma Surgeries, Gastrointestinal surgery and Endourology. He is a life member with the Association of Surgeons of India. 